\title{
CLASSICAL AND QUANTUM SYMMETRIES REDUCTION AND INTEGRABILITY
}

\author{
GIUSEPPE MARMO, GIOVANNI SPARANO AND GAETANO VILASI
}

Communicated by Gijs Tuynman

\begin{abstract}
Completely integrable systems always admit more alternative Hamiltonian descriptions. The geometrical formulation of quantum systems shows that similar conclusions hold true also for quantum systems. In addition, the description of quantum systems on Hilbert manifolds, e.g., the complex projective space, shows that not only quantum systems admit alternative Hamiltonian descriptions, they also admit alternative linear descriptions.
\end{abstract}

\section{Introduction}

In his Lectures on Dynamics [7], Jacobi starts with the problem of integrating the differential equations of motion. He explicitly says: In Mécanique Analytique one finds everything related to the problem of setting up and transforming the differential equations, but very little on their integration.

He goes on to elaborate what we nowdays call the Hamilton-Jacobi theory and elaborates on constants of the motion and symmetries.

The aim of our paper is to present a more general point of view in which the Hamilton-Jacobi theory is only an instance of the general procedure of integrating a system by reducing it to a normal form. In this respect we follow the view point of Birkhoff, all dynamical systems in the same orbit of the diffeomorphism group enjoy the same properties, therefore to study the integration problem one may select a particular representative of the equivalence class and consider it as a "normal form".

In this picture, the Hamilton-Jacobi procedure becomes a way to reduce a given Hamiltonian system to a normal form by replacing the full diffeomorphism group with the subgroup of canonical transformation necessary to achieve the transition to the normal form, the particular trnsformation is found by means of a generating function, solution of the Hamilton-Jacobi equation associated with the starting Hamiltonian functions, the comparison Hamiltonian and the one we want to tranform. 
By using the well known fact that Quantum Mechanics can be treated as a Hamiltonian system (on an infinite or finite dimensional manifold as the case may be), we maintain that most of our arguments apply both to Classical and Quantum Mechanics.

For Quantum Mechanics we should bear in mind that the carrier space (space of pure states) is the complex projective space $\mathbb{P H}$ associated to the Hilbert space $\mathbb{H}$. In this framework, the geometric structures pertinent to the standard treatment are a Riemannian structure, a Poisson structure and a complex structure. A compatibility condition among them qualifies the carrier space as a Kähler manifold. In this respect, i.e., from the point of view of geometric structures, quantum mechanics requires additional structures with respect to the symplectic structure of Hamiltonian classical dynamics. Of course the most difficult aspects of quantum mechanics have to do with the infinite dimensionality of the carrier space and the fact that infinite dimensional differential geometry is much less advanced than the finite dimensional one. The most serious problem in the generic infinite dimensional situation is that unbounded operators are not continuous, therefore all our assumptions about differentiability cannot be applied without further qualifications which should be made case-by-case.

Nevertheless, the structural aspects, what we may call synthetic as opposed to analytics will be essentially the same.

To avoid technicalities we shall mainly restrict to finite dimensional carrier spaces. To exhibit the variety of aspects emerging from the view point of Birkhoff we primarily deal with linear systems, the evolution associated with a differential equation will be our main concern, additional geometric structures on the carrier space will not be postulated at a "kinematical level" but derived by solving equations defined by the dynamics we start with.

By using symmetries and constants of the motion it is possible to derive nonlinear systems as reduction of linear ones. Of course, most of the properties valid for the linear situation will be inherited by the nonlinear one when they are compatible with the reduction procedure.

Many aspects will be illustrated by means of examples instead of formulating and proving general theorems. A more extensive treatment of these issues can be found in [4] and references therein.

Organization of the paper is as follows:

- The Geometrical Formulation of Quantum Mechanics

- Linear Systems on Vector Spaces (invariant structures: Poisson, symplectic, Lagrangian) 
- Bihamiltonian Systems

- From linear to nonlinear (what happens of the superposition rule, Riccati equation on $\mathbb{P H})$.

\section{Geometrical Formulation of Quantum Mechanics}

The observation that quantum theory could be described in the language of symplectic mechanics may be traced back in the work of Segal and Mackey $[8,11]$. More recently, several authors have been investigating the further developments of geometrical quantum mechanics, in doing so this methodology has unveiled new aspects and insights into the workings of the quantum world the way we understand it now, see [4] and references therein.

One particular aspect which may be connected with the existence of nonlinear transformations connecting linear descriptions seem to us worth of notice.

Let us start by reviewing very briefly how quantum mechanics is usually formulated.

With any physical system we associate a complex Hilbert space $\mathbb{H}$, the choice of a linear carrier space and a linear equation of motion is usually motivated [3] by the need to incorporate interference. The probabilistic interpretation of quantum mechanics requires the restriction to norm-one-vectors; to preserve the probability one usually restricts the evolution to be unitary, the differential equation of motion will then be associated with a skew-Hermitian "infinitesimal generator", written as i $H$ with $H$ Hermitian operator. Observables are identified with Hermitian operators which are usually thought of as the real elements of the $C^{*}$-algebra of bounded operators acting on $\mathbb{H}$.

If we use Dirac's bra and ket notation, we find that pure physical states are associated with rank-one projectors, this association depends on the specific Hermitian product we are using

$$
\rho_{\psi}=\frac{|\psi\rangle\langle\psi|}{\langle\psi \mid \psi\rangle}
$$

From the Schrödinger differential equation

$$
\frac{\mathrm{d}|\psi\rangle}{\mathrm{d} t}=\frac{H}{\mathrm{i} \hbar}|\psi\rangle
$$

one derives the equation of motion for rank-one projectors

$$
\frac{\mathrm{d} \rho_{\psi}}{\mathrm{d} t}=\frac{1}{\mathrm{i} \hbar}\left[H, \rho_{\psi}\right] .
$$


Using the linearity of the equation, it is possible to extend this equation to convex linear combinations

$$
\rho=\sum_{j} p_{j} \rho_{\psi_{j}}
$$

with $p_{j} \geq 0, \sum_{j} p_{j}=1$, to get the equation of motion on density states

$$
\frac{\mathrm{d} \rho}{\mathrm{d} t}=\frac{1}{\mathrm{i} \hbar}[H, \rho] .
$$

If we use also complex linear combinations we arrive at equations of motion for generic operators

$$
\frac{\mathrm{d} A}{\mathrm{~d} t}=\frac{1}{\mathrm{i} \hbar}[H, A]
$$

which represent equations of motion in the Heisenberg picture.

It is now clear that the dynamics on the complex projective space ceases to be linear, the sum of two rank-one projectors is no more a rank-one projector.

To remedy this situation, after all interference phenomena should be treated also on this nonlinear space, we have to write down a composition law for pure states which is inner, out of two pure states gives another pure state.

Such a composition law may be given the following form [4,9], out of $\varrho_{1}$ and $\rho_{2}$ we get

$$
\rho=\left|c_{1}\right|^{2} \rho_{1}+\left|c_{2}\right|^{2} \rho_{2}+c_{1} c_{2}^{*} \frac{\rho_{1} \rho_{o} \rho_{1}}{\sqrt{\operatorname{Tr}\left(\rho_{1} \rho_{o} \rho_{2} \rho_{0}\right)}}+\text { h.c. }
$$

with the understanding that $\left|c_{1}^{2}\right|$ and $\left|c_{2}^{2}\right|$ are required to satisfy $\rho^{2}=\rho, \operatorname{Tr} \rho=1$.

The reason we have inserted this composition law by means of fiducial state $\rho_{0}$ is due to the circumstance that, when written in homogeneous coordinates for the complex projective space, Schrödinger equation becomes a Riccati-type equation and the given composition is exactly the one we would get by composing solutions by means of the "harmonic ratio" [2]. To illustrate how Riccati equation emerges from Schrïidinger equation on the space of state vectors, we consider the two-dimensional case, $\mathbb{H}=\mathbb{C}^{2}$. Let us introduce an orthonormal basis $\left|e_{1}\right\rangle,\left|e_{2}\right\rangle$, $\left\langle e_{j} \mid e_{k}\right\rangle=\delta_{j k}$, and define associated coordinates, by setting $z_{1}(\psi)=\left\langle e_{1} \mid \psi\right\rangle$, $z_{2}(\psi)=\left\langle e_{2} \mid \psi\right\rangle$. In this basis we write Schrödinger equation as

$$
\frac{\mathrm{d}}{\mathrm{d} t}\left|\begin{array}{l}
z_{1} \\
z_{2}
\end{array}\right|=\frac{H}{\mathrm{i} \hbar}\left|\begin{array}{l}
z_{1} \\
z_{2}
\end{array}\right|
$$

whith $H$ being the Hermitian matrix

$$
\left|\begin{array}{ll}
h_{11} & h_{12} \\
h_{21} & h_{22}
\end{array}\right| .
$$


In this way we find

$$
\dot{z}_{1}=\frac{1}{\mathrm{i} \hbar}\left(h_{11} z_{1}+h_{12} z_{2}\right), \quad \dot{z}_{2}=\frac{1}{\mathrm{i} \hbar}\left(h_{21} z_{1}+h_{22} z_{2}\right)
$$

along the corresponding complex conjugate equations for $\bar{z}_{1}, \bar{z}_{2}$.

We introduce homogeneous coordinates to implement the probabilistic interpretation, according to which the physical state is associated with the ray defined by $\lambda\left|\begin{array}{l}z_{1} \\ z_{2}\end{array}\right|$ with $\lambda$ any complex number different from zero, we have $\xi=z_{1} / z_{2}$ and obtain

$$
\frac{\mathrm{d} \xi}{\mathrm{d} t}=\frac{h_{21}}{\mathrm{i} \hbar}+\frac{h_{22}-h_{11}}{\mathrm{i} \hbar} \xi-\frac{h_{12}}{\mathrm{i} \hbar} \xi^{2} .
$$

This Riccati equation has composition law

$$
\frac{\xi(t)-\xi_{1}(t)}{\xi(t)-\xi_{2}(t)}=k \frac{\xi_{1}(t)-\xi_{3}(t)}{\xi_{2}(t)-\xi_{3}(t)}
$$

in terms of solutions $\xi_{1}(t), \xi_{2}(t), \xi_{3}(t)$ and initial conditions determining $k$. This composition law says we can still describe interference phenomena even though the equation of motion is not linear. Perhaps, we should also remark that the lack of completeness of the vector field representing the evolution, equation of motion, is an artifact of the coordinates we have introduced to describe the space of pure states $S^{2}$. The actual dynamics is associated with a one-parameter group of transformations preserving the Kähler structure on the complex projective space $\mathbb{C P}^{1} \equiv S^{2}$. Schrödinger equation on $\mathbb{H}=\mathbb{C}^{n}$ may be written in a similar form

$$
\frac{\mathrm{d} \psi}{\mathrm{d} t}=\frac{H}{\mathrm{i} \hbar} \psi=\left|\begin{array}{cc}
H_{1} & V \\
V^{\dagger} & H_{2}
\end{array}\right| \psi \text {. }
$$

Separating the upper $n-1$ components of $\psi$ denoted by $\xi$ from the $n$-th one $\eta$ i.e., $\psi=\left|\begin{array}{c}\xi \\ \eta\end{array}\right|$, Schrödinger equation may be written as

$$
\begin{aligned}
\frac{\mathrm{d} \xi}{\mathrm{d} t} & =H_{1} \xi+V \eta \\
\frac{\mathrm{d} \eta}{\mathrm{d} t} & =V^{\dagger} \xi+H_{2} \eta .
\end{aligned}
$$

If we now set $z=\xi / \eta$, in analogy with the two-dimensional case, we get

$$
\dot{z}=V+H_{1} z-z H_{2}-z V^{\dagger} z .
$$

This is again a Riccati-type equation, but now $z$ ia an $(n-1)$-component vector, as is $V$, while $H_{2}$ is a single real variable. 
As the carrier space is no more linear, we cannot consider operators anymore, thus we shall replace them with expectation value functions

$$
e_{A}([\psi])=\frac{\langle\psi \mid A \psi\rangle}{\langle\psi \mid \psi\rangle}=\operatorname{Tr} \rho_{\psi} A .
$$

When the operator $A$ is hermitian these expectation value functions are called Kälherian functions. They are completely characterized by the property that the Hamiltonian vector fields associated with them are also Killing vector fields. Here the Poisson structure and the Riemannian tensors are those arising from projecting the imaginary and the real part of the Hermitian tensor from the complex Hilbert space to the space of rays, or complex projective space, when expressed in contravariant form.

In summary, the standard formulation of quantum mechanics, when written in terms of pure states gives rise to a geometrical formulation on the manifold of rays, the complex projective space. Equations of motion are described by a Hamiltonian vector field which is also a Killing vector field preserving the complex structure.

In conclusion the equations of motion of a quantum system are represented by a vector field $\Gamma$ on a Hilbert manifold $M$, for physical motivations connected with the probabilistic interpretation, the vector field is required to be Hamiltonian and Killing

\section{Classical Dynamics}

In principle it would be possible to formulate also classical dynamics on the Hilbert space of square integrable functions on phase space with the Liouville measure associated with the symplectic volume $\omega^{n}$. Now, infinitesimal generators of the evolution may be arbitrary differential operators of arbitrary order (indeed, they could even be pseudo-differential operators). The non local character of quantum mechanics is encoded in the non local product of expectation-value functions and the corresponding derivations associated with higher order differential operators. In classical mechanics, described on phase space, we may write the equations of motion in the form

$$
\mathrm{i} \frac{\partial f(q, p, t)}{\partial t}=\hat{H} f(q, p, t)
$$

with $\hat{H}$ the Liouville operator

$$
\hat{H}=-\mathrm{i} \frac{\partial H}{\partial p} \frac{\partial}{\partial q}+\mathrm{i} \frac{\partial H}{\partial q} \frac{\partial}{\partial p}
$$


and $H$ is the Hamiltonian function. In general, to restrict the equation to states, we replace $f(p, q)$ with $\rho(p, q)$ which is a probability density on phase space. It is possible to introduce the Hilbert space of square integrable functions on phase space with respect to the Liouville measure $\omega^{n}, \omega$ being the symplectic structure. the Liouville probability density may now be written as $\rho(p, q)=\psi^{*}(p, q) \psi(p, q)$. It should be stressed that in this picture the operators $\hat{q}$ and $\hat{p}$ commute differently than in quantum mechanics. all operators are first order operators while are derivations for the standard point-wise product and therefore their exponentiations will be one-parameter groups of automorphisms of the local point-wise product. The motivation for introducing this Hilbert space point of view for classical mechanics was to study the ergodicity issues and the understanding of Lyapunov exponents. This was attempted by Koopman [6].

In this picture, the one-parameter group of transformations is an automorphism group of the point wise product on functions, i.e., of the local product.

In the quantum case, the one-parameter group of "unitary transformations" on the complex projective space does not generate automorphisms of the point wise product of Kälherian functions but of the non local product corresponding to the operator product, namely

$$
e_{A} \star e_{B}([\psi])=\operatorname{Tr} \rho_{\psi} A \cdot B=e_{A B}([\psi]) .
$$

It should be remarked, however, that the requirement of irreducibility of the representation for the canonical commutation relations requires that quantum mechanics be formulated on the space of square integrable functions on a Lagrangian submanifold of the phase space of the classical system.

In any case, our considerations allow us to say that a classical dynamical system is represented by a vector field $\Gamma$ on a carrier space $M$ endowed with a symplectic structure $\omega$ such that $i_{\Gamma} \omega=-\mathrm{d} H$. Thus, unlike for quantum case, the vector field describing the dynamics is Hamiltonian but is not required to be a Killing vector field.

Now, we can consider the problem of integrating a dynamical vector field $\Gamma$ on a manifold $M$.

As Birkhoff [1] pointed out, all dynamical systems in the orbit of Diff $(M)$ passing through $\Gamma$ will share the same properties of $\Gamma$, therefore we might study a particularly relevant representative of the equivalence class to unveil the properties of each one of them in the orbit, what would be called a "normal form".

To be concrete, we shall carry on this analysis in the simple case of linear systems. 


\section{Linear Structures and Linear Vector Fields}

Our starting point is a pair $(\Gamma, M)$, a vector field $\Gamma$ representing the dynamics and a carrier manifold $M$, thought of as a space of states.

A linear structure on $M$ is characterized by a vector field $\Delta$ having the following properties:

1. There exists one and only one point, a critical point for $\Delta$, such that $\Delta\left(m_{0}\right)=0$

2. The eigenvalue problem

$$
L_{\Delta} h=0 . h
$$

has only trivial solutions on each connected component of $M$

3. The eigenvalue problem

$$
L_{\Delta} f=f
$$

has as many functionally independent solutions $\left(f_{1}, f_{2}, \ldots, f_{n}\right)$ as the dimension of the carrier space $M$

4. $\Delta$ is a complete vector field on $M$.

Such a vector field $\Delta$ is also called a dilation vector field.

Proposition 1. A connected manifold $M$, possessing a dilation vector field $\Delta$, may be endowed with a vector space structure.

The statement follows by selecting a family of independent solutions for the eigenvalue equation

$$
L_{\Delta} f=f
$$

say $\left(f_{1}, f_{2}, \ldots, f_{n}\right)$ with $f_{i} \in \mathfrak{F}(\mathrm{M})$. We may define a composition law on points of $M$ by setting

$$
\left(\lambda_{1} m_{1}+\lambda_{2} m_{2}\right)(f)=\lambda_{1} f\left(m_{1}\right)+\lambda_{2} f\left(m_{2}\right), \quad m_{1}, m_{2} \in M
$$

where $f$ belongs to the linear span of $\left(f_{1}, f_{2}, \ldots, f_{n}\right)$. Notice that $(\lambda \cdot m)(f)=$ $\lambda \cdot f(m)$ gives again a point in $M$ because of the completeness assumption on $\Delta$. By using $\left(f_{1}, f_{2}, \ldots, f_{n}\right)$ as a coordinate system for the whole of $M$, we have that $\mathrm{d} f_{j}(\Delta)=f_{j}$ implies $f_{j}\left(m_{0}\right)=0$ and $\Delta=f_{j} \partial / \partial f_{j}$.

Thus the point $m_{0}$ is the null vector of the vector space structure on $M$. It is now clear that any other vector field in the orbit $\operatorname{Diff}(M)$ passing through $\Delta$ will give rise to another linear structure. 
The subgroup of diffeomorphisms preserving $\Delta$ will be the group of linear transformations, $\mathrm{GL}(M, \Delta)$, which is isomorphic to $\mathrm{GL}(n, \mathbb{R})$ therefore alternate linear structures are parametrized by Diff $(M) / \mathrm{GL}(M, \Delta)$.

Having defined a linear structure, it is possible to define linear vector fields

Definition 2. A vector field $\Gamma$ is linear with respect to $\Delta$ if $[\Gamma, \Delta]=0$.

Proposition 3. Any linear vector field $\Gamma$ is represented by a class of matrices defining an orbit of $\mathrm{GL}(n, \mathbb{R})$.

Proof: Consider a particular independent set of solutions $L_{\Delta} f_{j}=f_{j}, j=1,2, \ldots, n$. We have

$$
L_{\Gamma} L_{\Delta} f_{j}=L_{\Gamma} f_{j}=\gamma_{j}^{k} f_{k}, \quad \gamma_{j}^{k} \in \mathbb{R} .
$$

If we choose a different basis of solutions, we associate a different matrix to $\Gamma$, all of them will be related by the matrix $\left\|T_{k}^{i}\right\|$ taking from one basis to another, therefore they are elements in the orbit $T \gamma T^{-1}$ with $T \in \operatorname{GL}(n, \mathbb{R})$. The vector field $\Gamma$ may be represented as a linear homogeneous differential operator

$$
\Gamma=f_{k} \gamma_{j}^{k} \frac{\partial}{\partial f_{j}} .
$$

The equation of motion associated with $\Gamma$ are written, in the selected basis,

$$
\frac{\mathrm{d} h}{\mathrm{~d} t}=f_{k} \gamma_{j}^{k} \frac{\partial h}{\partial f_{j}} .
$$

In finite dimensions, a solution is provided by the exponentiation of the representative matrix $\gamma$, i.e.,

$$
\vec{x}(t)=\mathrm{e}^{t \gamma} \vec{x}(0) .
$$

Even though for each initial condition we have found the solution, it is clear that many questions like conservation, stability, periodic orbits and many others cannot be easily answered from the given form.

As matter of fact more can be said if we reduce $\gamma$ to some normal form. For instance, we may use Schur decomposition to write $\gamma=N+S$, with $N$ a nilpotent matrix and $S$ semisimple, $[N, S]=0$.

The commutativity property gives rise to the "composition of independent motions"

$$
\mathrm{e}^{t N} \cdot \mathrm{e}^{t S}=\mathrm{e}^{t(N+S)}
$$


As $N$ is nilpotent, $\mathrm{e}^{t N}$ reduces to a polynomial in $t$, of degree equal one-less nilpotency index.

We may derive in general that the commutant of $\gamma$, say $[\gamma]^{\prime}$, the algebra of matrices commuting with $\gamma$ contains all powers of $\gamma$ and is Abelian if all eigenvalues are not degenerate. If $\gamma$ admits degenerate eigenvalues, the algebra of symmetries will not be Abelian.

An interesting question from our point of view is the existence of constants of motion.

From our experience coming from Hamiltonian formalism, where there is a connection between symmetries and constants of the motion, we could investigate the possibility that our dynamical vector field preserves quadratic forms or bilinear maps.

In geometrical languages solutions of the equation $L_{\Gamma} \mathcal{T}=0$ would give all invariant tensor field $\mathcal{T}$.

Of particular relevance are tensor fields of rank two, perhaps decomposed into symmetric and skewsymmetric ones.

For instance $\Gamma=\Lambda(\mathrm{d} H)$, i.e., the existence of an Hamiltonian description in terms of Poisson tensor $\Lambda$ and the quadratic hamiltonian $H$, when written in terms of matrices, would give $\gamma=\Lambda H$, where $\Lambda$ and $H$ are the representative matrices for the Poisson tensor and the Hamiltonian function respectively. We would have

$$
\Lambda=\Lambda_{j k} \frac{\partial}{\partial f_{j}} \wedge \frac{\partial}{\partial f_{k}}, \quad H=\frac{1}{2} H^{j k} f_{j} f_{k}
$$

so that at matrix level $\gamma_{j}^{k}=\Lambda_{j m} H^{m k}$.

Thus, the existence of Hamiltonian descriptions for the linearizable vector field $\Gamma$ amounts to the decomposition of the representative matrix as the product of a skew-symmetric times a symmetric matrix.

For the generic case we can immediately give a necessary and sufficient condition for such a decomposition.

Proposition 4. If $\gamma$ has minimal degeneracy, a necessary and sufficient condition for the decomposition $\gamma=\Lambda \cdot H$, is that all odd powers of $\gamma$ are traceless.

Proof: In one direction the choice is obvious because $\operatorname{Tr} \gamma=\operatorname{Tr} \gamma^{\dagger}$ and $(\Lambda \cdot H)^{\dagger}=$ $-H \cdot \Lambda$. The general proof may be found in [5].

What is relevant for our considerations is the part that all matrices in the orbit

$$
T \gamma T^{-1}=\gamma\left(T \Lambda T^{\dagger}\right)\left(T^{\dagger}\right)^{-1} H T^{-1}=\left(T \Lambda T^{\dagger}\right)\left(T^{-1}\right)^{\dagger} H T^{-1}
$$


have also the same property, i.e., they are Hamiltonian, factorizable with respect to different Poisson structures and different Hamiltonians.

Of course, of particular significance is the family of transformations for which $T \gamma T^{-1}=\gamma,\left(T \Lambda T^{\dagger}\right) \neq \Lambda$, i.e., symmetries for $\gamma$ which are not canonical.

These particular symmetries will carry one Hamiltonian description into an alternative one. We find that Hamiltonian systems admitting symmetries which are not canonical will always be bi-Hamiltonian.

From our considerations it should be clear that there are linear systems with a large group of symmetries which may admit no constants of the motion.

For instance this is the case for the dilation vector field $\Delta$ itself. The equation $L_{\Delta} h=0$ admits only trivial solutions while the symmetry group is the full $\mathrm{GL}(n, \mathbb{R})$.

A generic linear Hamiltonian system will have all powers $\gamma^{k}$ as symmetries but only the odd powers $\gamma^{2 k+1}$ will generate canonical symmetries. Thus, any linear Hamiltonian system always admits alternative Hamiltonian descriptions.

However, this way of generating alternative Hamiltonian descriptions will not exhaust the family of alternative ones. For instance the two-dimensional isotropic Harmonic Oscillator has different decompositions with $H$ positive definite (the standard one) or with signature $(++--)$ which arises from

$$
\Lambda=\frac{\partial}{\partial q_{1}} \wedge \frac{\partial}{\partial p_{1}}-\frac{\partial}{\partial q_{2}} \wedge \frac{\partial}{\partial p_{2}}, \quad H=\frac{1}{2}\left(p_{1}^{2}+q_{1}^{2}-p_{2}^{2}-q_{2}^{2}\right) .
$$

Obviously this factorization cannot be related to the standard one by similarity transformation.

Remark 5. When the Poisson tensor is not degenerate, we can invert it and define a symplectic structure. When this symplectic structure is exact, we can consider a symplectic potential and use it to define a cotangent bundle structure on M. From the Hamiltonian, if transversal to fibers, with a nondegenerate Hessian, we can go to the Lagrangian description. It is therefore clear that the chain of steps PoissonSymplectic-Lagrangian puts more and more restrictions on the dynamical vector field we are considering, and correspondingly on the transformation group we may use to find "normal forms".

A final comment is in order when the orbits of our dynamical evolution are all bounded. Going back to the decomposition of $\gamma=N+S$, it is clear that for bounded orbits $N$ must be zero and $S$ should admit only purely imaginary eigenvalues if they are not vanishing. 
Thus, not vanishing purely imaginary eigenvalues require $M$ to have even dimension. As a matter of fact, in this situation, $M$ maybe endowed with a complex structure so that it becomes isomorphic with $\mathbb{C}^{n}, \Lambda$ will represent the imaginary part of an Hermitian structure and in all we can prove the following proposition.

Proposition 6. A complex linear vector field $\Gamma$ generates a flow $\phi_{t}: \mathbb{C}^{n} \rightarrow \mathbb{C}^{n}$ preserving same Hermitian scalar product $h$, i.e., $\phi_{t}^{\star} h=h$, iff any one of the following equivalent conditions is satisfied.

1. $H=H^{\dagger}$, where the adjoint is taken with respect to the scalar product defined by $h$, i.e., $L_{\Gamma} h=0$

2. $H$ is diagonalizable and has a real spectrum

3. all orbits $\mathrm{e}^{-\mathrm{i} H t} \psi$ are bounded sets, for any initial condition $\psi$.

When moving to infinite dimensions, one may try to use a similar procedure, however the corresponding separation of $H$ holds true only for a special class of operators. Therefore one has to use a different approach. Further details can be found in [10].

\section{Acknowledgements}

We wish to thank the Italian Istituto Nazionale di Fisica Nucleare (INFN) and the Agenzia Spaziale Italiana (ASI) for partial support.

\section{References}

[1] Birkhoff G., Dynamical Systems, Am. Math. Soc., Providence 1927.

[2] Carinena J., Gracia X., Marmo G., Martinez E., Munoz-Lecanda M. and Roman-Roy N., Geometric Hamilton-Jacobi Theory, Int. J. Geom. Meth. Mod. Phys. 3 (2006) 1417-1458.

[3] Dirac P., The Principles of Quantum Mecahanics, Clarendon Press, Oxford 1958.

[4] Ercolessi E., Marmo G. and Morandi G., From the Equations of Motion to the Canonical Commutation Relations, La Rivista del Nuovo Cimento, Serie V 33 (2010) 401-590.

[5] Giordano M., Marmo G. and Rubano C., The Inverse Problem in Hamiltonian Formalism: Integrability of Linear Hamiltonian Fields, Inverse Problems 9 (1993) 443-467. 
[6] Koopman B., Hamiltonian Systems and Transformations in Hilbert Space, Proc. Natl. Acad. Sci. 17 (1931) 315-318.

[7] Jacobi C., Jacobi's Lectures on Dynamics (delivered at the University of Konigsberg in the Winter Semester 1842-1843 and According to the Notes Prepared by C. Brockardt), Hindustan Book Agency, 2009.

[8] Mackey G., Mathematical Foundations of Quantum Mechanics, Benjamin, New York 1963.

[9] Man'ko V., Marmo G., Sudarshan E. and Zaccaria F., Inner Composition Law of Pure States as a Purification of Impure States, Phys. Lett. A 273 (2000) 3136.

[10] Marmo G., Simoni A. and Ventriglia F., Quantum Systems and Alternative Unitary Descriptions, Int. J. Mod. Phys. A 19 (2004) 2561-2578.

[11] Segal I., Mathematical Problems of Relativistic Physics, J. Math. Phys. 1 (1960) 468-488.

Giuseppe Marmo

Dipartimento di Scienze Fisiche

Università degli Studi di Napoli

Istituto Nazionale di Fisica Nucleare

Napoli 80126, ITALY

E-mail address: marmo@na. infn. it

Giovanni Sparano

Dipartimento di Matematica

Università degli Studi di Salerno

Istituto Nazionale di Fisica Nucleare

Fisciano 84084, ITALY

E-mail address: sparano@unisa. it

Gaetano Vilasi

Dipartimento di Fisica

Università degli Studi di Salerno

Istituto Nazionale di Fisica Nucleare

Fisciano 84084, ITALY

E-mail address: vilasi@sa.infn. it 